\title{
Présentation de compte-rendu d'ateliers sur des maladies à transmission vectorielle émergentes et les milieux urbains
}

\author{
Karmali $\mathrm{M}^{1 *}$, Weinstock $\mathrm{D}^{1}$ \\ Affiliation \\ ${ }^{1}$ Institut des politiques sociales et de la santé de I'Université McGill, Montréal (Québec) \\ *Correspondance : mkarmali@rogers.com
}

Citation proposée : Karmali M, Weinstock D. Présentation de compte-rendu d'ateliers sur des maladies à transmission vectorielle émergentes et les milieux urbains. Relevé des maladies transmissibles au Canada 2016;42:220. https://doi.org/10.14745/ccdr.v42i10a01f

\section{Introduction}

Les villes du monde entier enregistrent une croissance spectaculaire en taille et en population et sont devenues des points de convergence dans le cadre de la mondialisation, notamment pour le déplacement rapide des personnes, des aliments, des marchandises et des maladies contagieuses entre les frontières. Les épidémies transmises par les moustiques persistants et en expansion causées par le virus de la dengue, le virus Chikungunya et le virus Zika sont particulièrement préoccupantes. Ces éclosions peuvent être davantage exacerbées par le changement climatique et de mauvaises conditions sociales, en particulier dans les pays à revenu faible et intermédiaire. Au Canada, les principales maladies à transmission vectorielle (MTV) sont la maladie de Lyme, qui est transmise par les tiques, et l'infection par le virus du Nil occidental, qui est transmise par les moustiques. Ces maladies sont également observées en milieu urbain et leur incidence est influencée par le réchauffement climatique.

Dans de nombreux pays, les épidémies de maladies chroniques telles que l'obésité, le diabète de type 2, les maladies cardiovasculaires et respiratoires ont eu une incidence négative sur la santé des populations urbaines au cours des dernières décennies. Pour lutter contre cela, la planification urbaine contemporaine au Canada et dans d'autres pays développés a mis l'accent sur la réduction de l'étalement urbain et suburbain et sur la promotion d'un paysage urbain " plus vert » et « plus bleu » contenant mois d'automobiles et un air plus pur. L'objectif est de permettre aux personnes de davantage marcher et faire du vélo et de moins prendre la voiture, ce qui permet de réduire la pollution et de préserver le milieu naturel périurbain.

Toutefois, nous ne savons pas dans quelle mesure l'expansion continue du milieu bâti (c.-à-d. du milieu urbain et suburbain) et les efforts visant à promouvoir un mode de vie plus sain peuvent influencer de façon involontaire la survie et la reproduction des moustiques et des tiques ainsi que l'exposition des personnes à ceux-ci. Cette exposition pourrait découler de l'établissement d'un paysage plus vert et plus bleu, de la présence de bassins d'eau stagnante dans le milieu urbain, et de l'effet d'« îlot de chaleur " par lequel les températures dans les villes sont bien plus élevées que dans les zones environnantes. Ces effets peuvent être davantage exacerbés par le changement climatique. De plus, les densités de population urbaine accrues et l'arrivée dans les villes de voyageurs et de migrants infectés font augmenter les risques de transmission des virus par les moustiques.

Afin d'étudier cette question, un atelier multidisciplinaire et multisectoriel organisé par I'Institut des politiques sociales et de la santé de I'Université McGill a eu lieu les 25 et 26 avril 2016. L'objectif de l'atelier était d'étudier les MTV émergentes et les effets du changement climatique, des stratégies de planification urbaine pour un mode de vie plus sain, et des stratégies d'atténuation des MTV émergentes. Les conférenciers et les participants comprenaient des experts et des responsables des politiques dans les disciplines suivantes : santé publique, maladies infectieuses, épidémiologie, santé et politique sociale, planification urbaine, économie, écologie, sociologie, ingénierie et climatologie. Sur les deux jours, il y a eu des présentations officielles faites par des experts et un dialogue interdisciplinaire dans le but de déterminer les stratégies d'atténuation et les lacunes en matière de recherche en ce qui concerne les défis émergents que présentent les MTV dans les villes canadiennes, dans le contexte du changement climatique et d'un mode de vie urbain sain.

Dans ce numéro de la revue, nous présentons des résumés des documents présentés, puis un résumé des présentations en atelier, des discussions et des prochaines étapes, notamment des questions de recherche visant à faire progresser ce domaine d'enquête.

Les MTV émergentes et le changement climatique représentent des menaces réelles pour le bien-être futur des Canadiens, notamment de ceux qui vivent en ville. Cet atelier fournit une introduction et une impulsion pour que les experts et les responsables des politiques de différents secteurs et de différentes disciplines étudient les approches à multiples facettes qui seront nécessaires pour relever ce défi.

\section{Conflit d'intérêts}

Aucun. 